\title{
APORTACIÓN AL CONOCIMIENTO DEL CICLO BIOLÓGICO DE CHROMULINA NEVADENSIS
}

\author{
Presentación CARRILLO, Luis CRUZ PIZARRO \& Pedro M. SÁNCHEZ \\ CASTILLO
}

\begin{abstract}
RESUMEN: En este trabajo se amplía y se comenta la descripción de Chromulina nevadensis P.M. Sánchez. El estudio se realiza en una laguna oligotrófica de alta montaña (La Caldera), próxima a la laguna de Aguas Verdes, donde se obtuvo el material para su descripción original. Igualmente realizamos algunas consideraciones sobre el comportamiento mixotrófico de este taxon, ya conocido para otros miembros de las crisofíceas. Para finalizar describimos, por primera vez, la morfología a MEB de su estatospora.
\end{abstract}

Palabras clave: Chromulina, Mixotrofia, Fitoplancton, Estatosporas

ABSTRACT: In this paper we present data about enlargement of the original description of Chromulina nevadensis. This study has been carried out on an oligotrophic high mountain lake (La Caldera) close to the lake where it was described (Aguas Verdes). Also we make some consideration about the mixotrophy, a nutritional behavior previously knew for other chrysophyceae taxa. Finally we study, by the first time, the morfology of its statospores with SEM.

Key words: Chromulina, Mixotrophy, Phytoplankton, Statospores.

\section{INTRODUCCIÓN}

Las crisofíceas constituyen uno de los grupos de protistas más interesantes desde distintas aproximaciones: taxonómica, ecológica, filogenética, etc. Sus representantes unicelulares son, por su simplicidad, excelentes modelos celulares para el estudio de diversos aspectos poco conocidos, cuyos resultados están provocando, por fin, nuevas aportaciones al conocimiento taxonómico y ecológico del grupo, ya que como indica Sandgren (1989), las crisofíceas, por diversas razones no han participado de los recientes estudios que sobre la biología del fitoplancton se han realizado en los últimos 25 años, permaneciendo aún como un grupo bastante desconocido. 
En cuanto a su ecología, constituyen un grupo de organismos ampliamente representado en agua dulce. Sus especies planctónicas, las más numerosas, están bien adaptadas a ecosistemas árticos y de alta montaña. Esto se hace especialmente patente en el género Chromulina, especialmente estudiado por autores que se centran en este tipo de ambientes (Skuja, 1964; Ettl, 1968; etc).

\section{MATERIAL Y MÉTODOS}

EL material estudiado procede del estudio intensivo de un ciclo anual realizado durante el año 1986 en la laguna de alta montaña de La Caldera (Sierra Nevada, Granada). Los muestreos se realizaron durante el período libre de hielo y en la zona limnética de la laguna. Las muestras de fitoplancton se fijaron, en botellas de $100 \mathrm{ml}$, con lugol acético. Su estudio se realizó en microscopio invertido. La metodología para la realización de los análisis químicos y de pigmentos es la misma que la expuesta en Sánchez Castillo \& al. (1989).

\section{RESULTADOS Y DISCUSIÓN}

A. Variabilidad morfológica.

La descripción original de este taxon se llevó a cabo sobre ejemplares recolectados de forma esporádica en 3 pequeñas lagunas: Aguas Verdes, Virgen Media y Virgen Superior. El estudio intensivo de la laguna de La Caldera, nos ha permitido poner de manifiesto la existencia de un amplio espectro de variabilidad morfológica. Debido a la escasa bibliografía existente sobre el tema, estos cambios morfológicos apenas están documentados.

En este trabajo se amplía el rango de variabilidad morfológica descrita por Sánchez Castillo (1987), quien lo establecía en (6-)7-8(-9) x 4-5 $\mu \mathrm{m}$. Uno de los hechos más llamativos observados a lo largo del período libre de hielo, es la disminución de tamaño que sufre este taxon desde principio a final de ciclo (Figs. 1A1C).

La figura 2A muestra los cambios de densidad celular y biomasa que sufre $C$. nevadensis durante el período estudiado. A mediados de julio se produce el máximo poblacional de este taxon $(4000 \mathrm{cel} / \mathrm{ml})$ fo mado fundamentalmente por células bien desarrolladas, lo que produce un máximo de biomasa de $15000 \mu \mathrm{m} 3$, cuando el tamaño celular es grande. A partir de esta época, cuando las células pequeñas son mayoritarias (Fig. 2A), disminuyen considerablemente los valores de biomasa. Es igualmente notable la disminución que sufre la concentración de clorofila de la comunidad fitoplanctónica durante el ciclo de estudio (Fig. 2B), más aún si tenemos en cuenta que a partir de agosto aparece Cyanarcus sp., el otro taxon que domina el fitoplancton de esta laguna. 

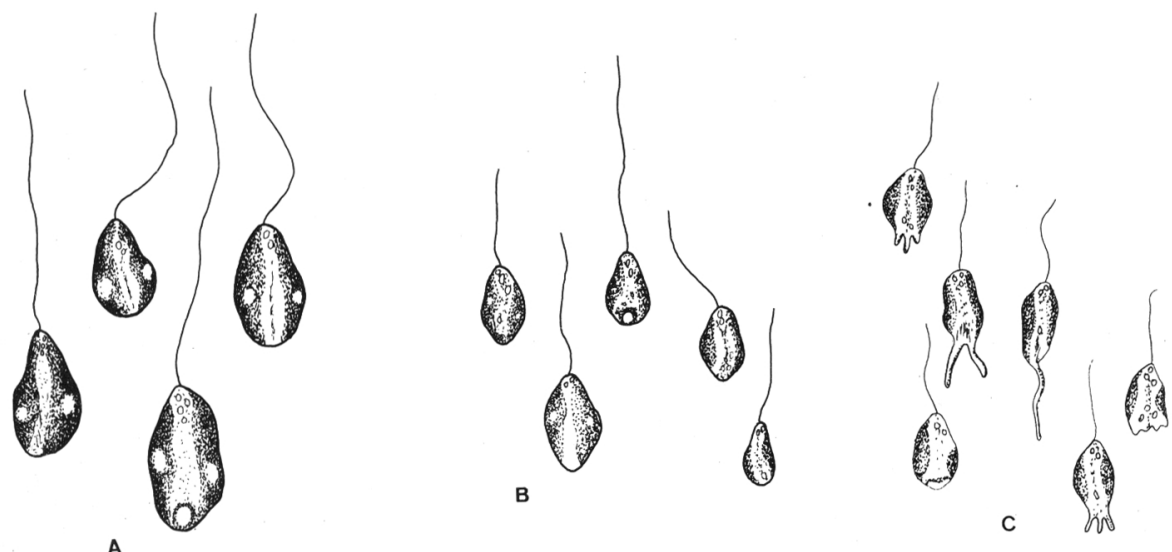

Figura 1.- Variabilidad morfológica de C. nevadensis durante el ciclo de estudio; A: Células de principio de ciclo con cloroplastos y pirenoides bien desarrollados. B: Células de menor tamaño y con pirenoides menos desarrollados o ausentes. C: Células con pseudópodos en el polo antiapical, sin pirenoides y con plastos claramente reducidos.

\section{A CHROMULINA NEVADENSIS}

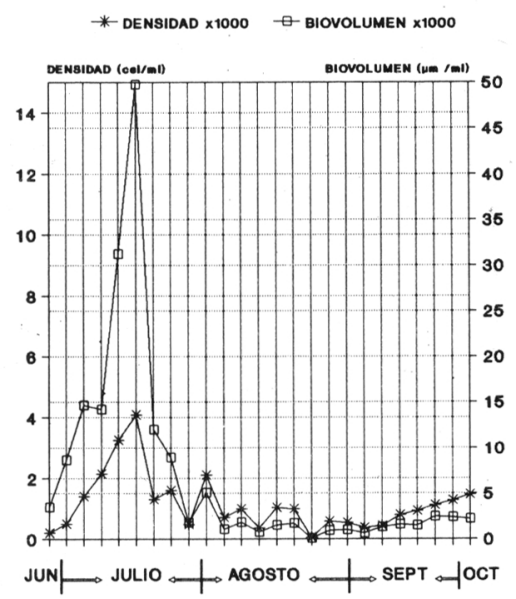

B CLOROFILA

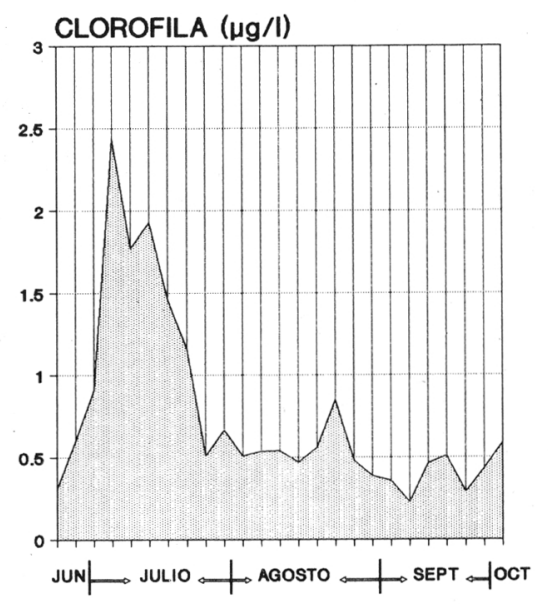

Figura 2.- A: Evolución, a lo largo del ciclo anual, de los valores de densidad celular y biovolumen. B: Evolución de la concentración de clorofila, de la comunidad fitoplanctónica, a lo largo del período de estudio. 
La disminución del tamaño celular suele ir acompañada por la del flagelo, las dimensiones observadas durante esta época oscilan entre: 3-5(-6) x 2-3 $\mu \mathrm{m}$, alcanzando el flagelo la longitud de la célula. De forma paralela se observa que las células de menor tamaño: 3-4 x 2-3 $\mu \mathrm{m}$ muestran unos cloroplastos muy reducidos, y en ocasiones sin pirenoides (Fig. 1B), los cuales están claramente diferenciados en las células de mayor tamaño (Fig. 1A). La reducción de los cloroplastos, así como la pobreza de ortofosfatos del medio en estos momentos (no detectables), hacen pensar en la posibilidad de nutrición mixotrófica para la supervivencia de este taxon hasta el final de período libre de hielo. La mixotrofía, carácter que ha sido detectado en varios miembros de las crisofíceas (Droop, 1974), en unas ocasiones es puesta de manifiesto por la existencia de pseudópodos (fagotrofia), mientras que en otras no es observable (osmotrofia).

\section{B. HETEROTROFIA}

La fuerte oligotrofia de las aguas de las lagunas de Sierra Nevada puede favorecer la existencia de aquellos organismos que tengan una capacidad potencial de nutrición mixotrófica. Este podría ser uno de los motivos del predominio de las crisofíceas en los ambientes oligotróficos.

El proceso de captura de material particulado por parte de los flagelados es muy variable. Entre las crisofíceas se ha observado que en Ochromonas parece facilitarse por el movimiento de los flagelos, el cual aproxima la partícula a la superficie de la célula. En Chromulina no se ha documentado esta aproximación del alimento, pero el cambio de longitud del flagelo antes comentado puede tener algún significado respecto a esta posibilidad. Por el contrario sí existe documentación en este género sobre la existencia de prolongaciones citoplasmáticas (pseudópodos) en el polo antiapical de las células (Ettl, 1968; Skuja, 1956, 1964): Chromulina dalecarlica Skuja, C. flavicans (Ehrnb.) Bütschli, C. frigidophila Skuja y C. skujae Starmach (Fig. 3).

En el estudio de C.nevadensis hemos realizado observaciones semejantes (Fig. 1C) en las poblaciones de pequeño tamaño que se desarrollan a partir de agosto, mostrando, desde un polo antiapical con 1, 2, 3 ó 4 pseudópodos bien desarrollados, pudiendo alcanzar la longitud de la célula, hasta numerosas y pequeñas evaginaciones que dan un aspecto aserrado al final de la célula. Estos pseudópodos nunca se han observado en células con cloroplastos y pirenoides bien desarrollados, por lo que parece clara la función de la fagotrofia como fuente complementaria de carbono en situaciones de fuerte oligotrofia.

La no excepcionalidad de la fagocitosis como tipo de nutrición en las crisofíceas se pone de manifiesto en las observaciones realizadas por numerosos autores: Aaronson (1973), Cole \& Wynne (1974) en Ochromonas, Bird \& Kalff (1978) y Sanders \& Porter (1988) entre otros en Chromulina. 


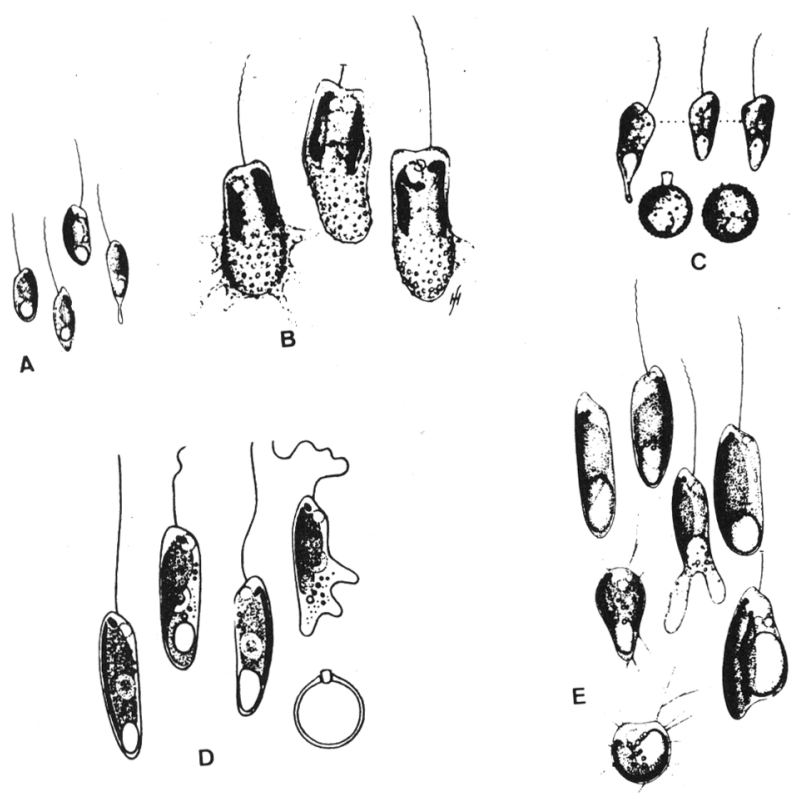

Figura 3.- Presencia de pseudópodos en distintas especies de género Chromulina.- A: $C$. delacarlica, B: C. flavicans, C: C. frigidophila, D y E: C. skujae.

La inducción de la fagotrofia en $C$. nevadensis pensamos que está determinada por la extrema oligotrofia del medio que habita, potenciándose quizás con el incremento de la temperatura como observa Aaronson (1974) en Ochromonas danica.

A pesar de los escasos conocimientos que aún tenemos sobre la importancia de la fagotrofia en el suministro de carbono a la célula en estos organismos, recientes trabajos, como el de Bird \& Kalff (1987) ponen de manifiesto que el 50\% del carbono que asimilan algunos de ellos (Dinobryon) tiene su origen en la fagotrofia. Debido al pequeño tamaño de $C$. nevadensis pensamos que deben ser las bacterias la principal fuente de alimentación fagotrófica. Otros táxones mejor estudiados, como los de Dinobryon son capaces de consumir más bacterias de la columna de agua que crustáceos, rotíferos y ciliados juntos (Sanders \& Porters, 1988), por lo que se hace evidente el papel de las crisofíceas en la regulación de la comunidad microbiana.

\section{ENQUISTAMIENTO}

En la mayoría de las algas planctónicas de agua dulce, el enquistamiento suele ser un mecanismo rápido que responde a una o a un conjunto de variables ambientales 
y que coincide con la disminución brusca o desaparición de la población. Por el contrario en la crisofíceas la formación de las estatosporas parece ser un mecanismo generalizado que ocurre a lo largo del desarrollo normal de la población, correspondiendo el incremento de las células vegetativas con el de las estatosporas. Este tipo de enquistamiento ha sido denominado «enquistamiento intrínseco» por Sandgren \& Flanangin (1986).

En nuestro caso no se cumple totalmente esta situación, ya que las estatosporas son observadas normalmente durante la primera parte del ciclo de estudio, coincidiendo con la población fototrófica. Quizás la poca concentración de silicatos reactivos que presenta la laguna de La Caldera, la menor de entre las estudiadas (Sánchez Castillo et al., 1989), sea el factor limitante para la formación de las estatosporas ya que, como se observa en la figura 4, el consumo de sílice en la laguna coincide con la formación de las estatosporas, produciéndose una notable reducción en este elemento. En estos momentos no existe en el medio ningún otro organismo con requerimientos de sílice para su metabolismo, por lo que deben ser las estatosporas las únicas responsables de esta disminución.

En la figura 5 se observa la morfología y ornamentación de la estatospora de C. nevadensis, observándose esférica (Fig. 5A) y totalmente cubierta de espinas truncadas en su ápice. El cuello (Fig. 5B) se observa formado por la fusión de las espinas que rodean a la apertura.

\section{A SILICATOS}

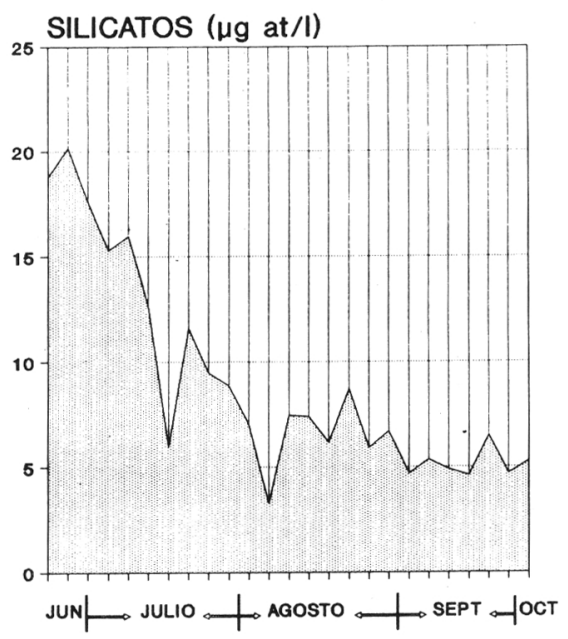

\section{B ESTATOSPORAS}

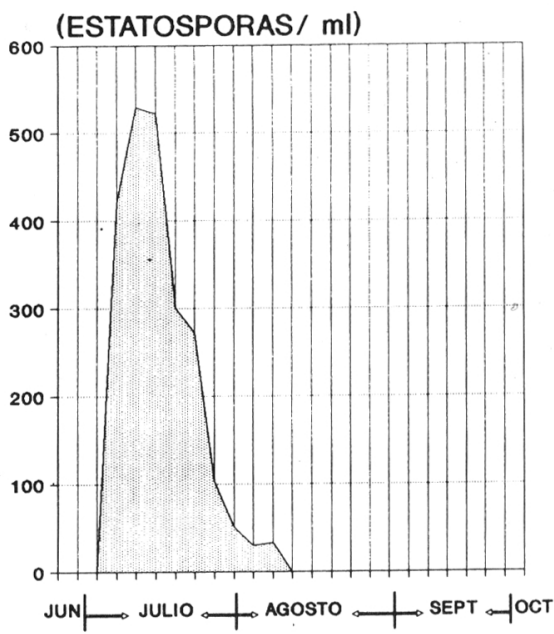

Figura 4.- Evolución de la concentración de silicatos y de la aparición de las estatosporas en el medio a lo largo del período estudiado. 


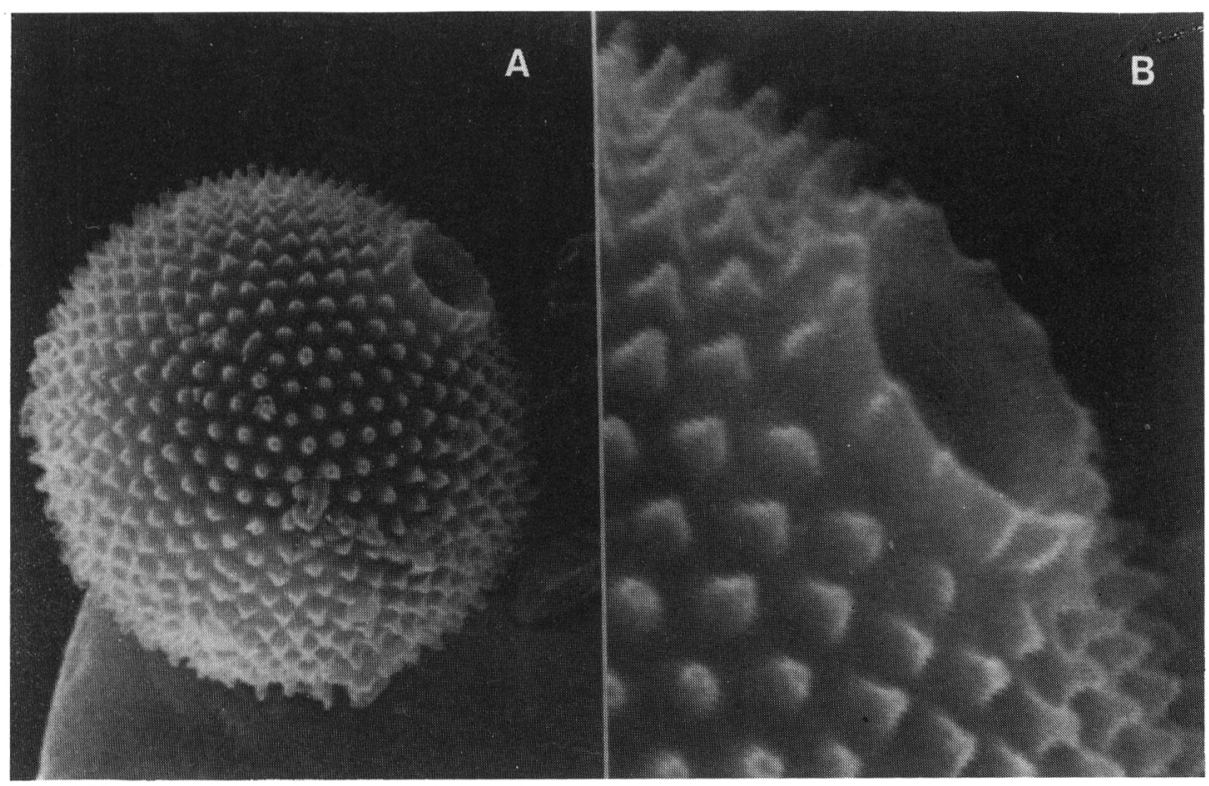

Figura 5.- Morfología de la estatospora. A: aspecto general; B: detalle de la apertura.

\section{BIBLIOGRAFÍA}

AARONSON, S. -1973- Particle aggregation and phagotrophy by Ochromonas. Arch. Mikrobiol., 92:39-44.

AARONSON, S. -1974- The biology and ultrastructure of phagotrophy in Ochromonas danica. J. Gen. Mikrobiol., 83:21-29.

BIRD, D.F. \& J. KALFF -1987- Algal phagotrophy: regulating factors and importance relative to photosinthesis in Dinobryon. Limnol. Oceanogr, 32:277-284.

COLE, G.T. \& M.J. WINNE -1974- Endocytosis of Microcystis aeruginosa by Ochromonas danica. J. Phycol., 10:397-410.

DROOP, M.R. -1974- Heterotrophy of Carbon. In Sterwart (ed.): Algal physiology and biochemistry. Blackwell Scientific Books.

ETTL, H. -1968-Ein Beitrag zur Kennitnis der Algenflora Tirols. Ber.nat.-med. ver. Innsbruck, 56:177-354.

SÁNCHEZ CASTILLO, P.M. -1987- Un nuevo taxon del género Chromulina: C. nevadenis. P.M. Sánchez. Cryptogamie. Algologie, 8(4):235-240.

SÁNCHEZ CASTILLO, P.M., L. CRUZ PIZARRO \& P. CARRILLO -1989-Caracterización del fitoplancton de las lagunas de alta montaña de Sierra Nevada y su relación con las características físico-químicas del medio. Limnetica, 5:37-50.

SANDERS, R.W. \& K.G. PORTER -1988- Phagotrophic phytoflagellates. In: K.C. Marshall. Advances in microbial ecology, 10:167-192.

SANDGREN, C.D. -1989-Growth and reproductive strategies of freshwater phytoplankton. 
Cambridge University Press. Cambridge.

SANDGREN, C.D. \& J. FLANANGIN -1986- Heterothallic sexuality and density dependt encystment in the chrysophycean alga Synura petersenii. J. Phycol., 22:206-216.

SKUJA, H. -1956- Taxonomische und biologische studien uber das phytoplankton schwedischer binnengewasser. Nova Acta Regiae Soc. Sci. Upsal., 16(3):1-400.

SKUJA, H. -1964- Gunzungen der algenflore und algenvegetation der Fjeldgengenden um Abisko. Nova Acta Regiae Soc. Sci. Upsal., 18:1-465.

(Aceptado para su publicación en Junio de 1.990)

Dirección de los autores: P. CARRILLO \& L. CRUZ PIZARRO: Departamento de Biología Animal, Ecología y Genética. Facultad de Ciencias. Universidad de Granada. 18001 Granada. P.M. SÁNCHEZ CASTILLO: Departamento de Biología Vegetal. Facultad de Ciencias. Universidad de Granada. 18001 Granada. 